\title{
Rapid Progressive Disease After Nivolumab Therapy in Three Patients with Metastatic Renal Cell Carcinoma
}

\author{
YUKI KOBARI $^{1}$, TSUNENORI KONDO ${ }^{1}$, TOSHIO TAKAGI ${ }^{2}$, KENJI OMAE ${ }^{2}$, \\ HAYAKAZU NAKAZAWA ${ }^{1}$ and KAZUNARI TANABE ${ }^{2}$ \\ ${ }^{1}$ Department of Urology, Tokyo Women's Medical University Medical Center East, Tokyo, Japan; \\ ${ }^{2}$ Department of Urology, Tokyo Women's Medical University, Tokyo, Japan
}

\begin{abstract}
Background/Aim: Rapid progressive disease $(R P D)$, accelerated tumour growth immediate after the initiation of immune checkpoint inhibitor therapy, has been reported in melanoma and lung cancer. Herein, we describe 3 cases of RPD during the initial phase of nivolumab treatment for metastatic renal cell carcinoma. Patients and Methods: The first and second patients received nivolumab in the fourth-line setting. The third patient received nivolumab therapy as third-line treatment. Results: The first patient developed severe respiratory failure due to carcinomatous lymphangiosis 14 days after initiation of nivolumab therapy. The second patient developed leg paraplegia due to rapid growth of the metastatic tumour at the sixth thoracic vertebrae 5 days later. The third patient developed grade 4 hypercalcemia due to RPD on day 3. Conclusion: Clinicians should be aware of RPD during the initial phase of nivolumab therapy, especially in patients with critical lesions in the late-line setting.
\end{abstract}

Nivolumab, an anti-programmed cell death 1 (PD-1) monoclonal antibody, was recently approved as a sequential therapy for advanced or metastatic renal cell carcinoma (RCC) because of the associated longer overall survival duration and lower incidence of grade 3 and 4 adverse events than that achieved with everolimus in the Checkmate 025 clinical trial (1). Anti-PD-1/anti-programmed death ligand-1 antibodies infrequently induce initial tumour growth

This article is freely accessible online.

Correspondence to: Tsunenori Kondo, MD, Ph.D., Department of Urology, Tokyo Women's Medical University Medical Center East, 2-1-10, Nishiogu, Arakawa-ku, Tokyo 116-8567, Japan. Tel: +81 338101111, Fax: +81338101110, e-mail: tkondo@twmu.ac.jp

Key Words: Central nervous system, disease progression, programmed cell death 1 ligand 1, renal cell carcinoma. followed by subsequent tumour regression in metastatic melanoma and lung cancer, which is commonly known as pseudoprogression (2-4). Progressive disease without subsequent regression has been observed during the initial phase of this new treatment for several cancers. We defined this phenomenon as rapid progressive disease (RPD). Herein, we describe three cases of RPD just after nivolumab therapy was administered for metastatic RCC.

\section{Case Report}

Case 1. A 63-year-old man was diagnosed as having left RCC accompanied by left adrenal and multiple lung metastases in 2011 for which he underwent cytoreductive nephrectomy with adrenalectomy (Table I). Pathological findings indicated clear cell RCC, G3. Interferon- $\alpha$ treatment was initiated postoperatively, and the patient showed a good initial response. He developed pancreatic metastasis and received pazopanib $(800 \mathrm{mg}$ ) daily in September 2014. In September 2016, pazopanib was switched to axitinib $(10 \mathrm{mg})$ daily because of progression of the pancreas metastases and multiple liver metastases. Since the liver and pancreatic metastasis rapidly worsened, he received nivolumab $(3 \mathrm{mg} / \mathrm{kg})$ in December 2016 (Figure 1a). Fourteen days later, he presented to the emergency room by ambulance because of the acute onset of dyspnoea.

We considered interstitial pneumonia, an immune-related adverse event induced by immune checkpoint inhibitors, on the basis of the presence of fine crackles and computed tomography (CT) evidence; therefore, treatment with pulse methylprednisolone at $500 \mathrm{mg}$ was initiated (Figure 1b). Upon admission, his lactate dehydrogenase level (418 U/l) and surfactant protein D level (174 ng/l) were slightly higher than normal, but the Krebs von den Lungen-6 level was within the normal range. Three hours after hospitalisation, his respiratory condition rapidly deteriorated, which necessitated intubation. Two hours after intubation, he developed sudden cardiopulmonary arrest, but fortunately, 
Table I. Patients' characteristics.

\begin{tabular}{lccc}
\hline & Case 1 & Case 2 & Case 3 \\
\hline Gender & Male & Female & Female \\
Age (years) & 63 & 66 & 71 \\
Initial tumour & Left RCC & Left RCC & Right RCC \\
Pathology & Clear cell pT3a & Clear cell pT3a & Clear cell pT3a \\
Metastases & Lung & Lung & Lung \\
& Liver & Liver & Kidney (left) \\
& Adrenal gland & Boncreas & Lymph node \\
Treatment & Pancreas & Thyroid gland & Sunitinib \\
First line & & & Axitinib \\
Second line & INF- $\alpha$ & Pazopanib & Nivolumab \\
Third line & Pazopanib & Sorafenib & - \\
Fourth line & Axitinib & Axitinib & 3 days \\
Disease onset after nivolumab therapy & Nivolumab & Nivolumab & Death \\
Outcome & 14 days & Deays & Complete paralysis \\
\hline
\end{tabular}

RCC, Renal cell carcinoma; INF, interferon.

our resuscitation attempts were successful. His condition temporary improved after resuscitation and steroid pulse therapy. He was ex-tubated 18 days after nivolumab treatment. Since he had normal cardiac function and interstitial pneumonia marker findings, carcinomatous lymphangiosis due to disease progression was suspected as the cause of the acute respiratory failure (Figure 1c). However, his general condition worsened, and he died of disease 42 days after nivolumab was administered.

Case 2. A 66-year-old woman with left RCC metastasised to the lung, liver, and bone underwent cytoreductive nephrectomy in 2014 (Table I). Pathological findings showed clear cell RCC G3 pT3a. Pazopanib treatment was initiated in June 2015. However, this treatment was discontinued because of severe diarrhoea; therefore, pazopanib was switched to sorafenib in September 2015. A new metastatic lesion was detected at the sixth vertebrae, which required us to switch her treatment from sorafenib to axitinib. During this third-line treatment, the metastatic tumour of the sixth vertebrae gradually increased in size and compressed the spinal cord. In January 2017, she began to experience numbness in both legs; therefore, treatment was switched to nivolumab $(3 \mathrm{mg} / \mathrm{kg})$. Five days after the administration of nivolumab, she developed paraplegia, that required for decompressive operation. Despite surgical treatment, the paraplegia did not improve, and she was put on the best supportive care.

Case 3. A 71-year-old woman with a medical history of right radical nephrectomy for RCC in 2001 developed multiple tumours in the left kidney (Table I). Seven months after partial nephrectomy with diagnosis of clear cell RCC G3, she developed lung metastasis. Metastasectomy was performed twice, but new metastases were detected in the left kidney and pancreas. Thus, we initiated treatment with sunitinib in July 2014. We switched sunitinib to axitinib (10 mg daily) in April 2016 because of progression of the left kidney tumour and the development of a right thyroid metastatic tumour. However, the lung, left kidney, and mediastinal lymph node metastasis progressed. Thus, nivolumab treatment was initiated in December 2016 as a third-line therapy. Three days after the first course of nivolumab, she was admitted to the emergency department for consciousness disorder. Hypercalcemia (calcium level, $16.3 \mathrm{mg} / \mathrm{dl}$ ) was found. Although the calcium level improved after treatment, the lung metastases progressed rapidly thereafter. She died of the disease 40 days after nivolumab treatment was initiated.

\section{Discussion}

During immune-checkpoint inhibitor therapy, tumors initially increase in size with a subsequent decrease of the tumour burden. This phenomenon is termed pseudoprogression. According to Chiou and Burotto, pseudoprogression has been reported in $6.7-12 \%$ of patients with malignant melanoma treated with immune checkpoint inhibitors (4). The incidence of drug discontinuation is currently unexpectedly higher than previously thought during the initial phase of anti-PD-1 treatment owing to severe adverse events or rapid tumour growth. Kanai et al. reported that nivolumab therapy was discontinued in $37 \%$ of patients with non-small cell lung cancer within 3 courses of treatment due to severe tumourrelated events. The most frequent event leading to treatment discontinuation was exacerbation of neurogenic symptoms, which occurred in patients with central nervous system metastases (5). Champiat et al. reported that $9 \%$ of patients 
$\mathbf{a}$

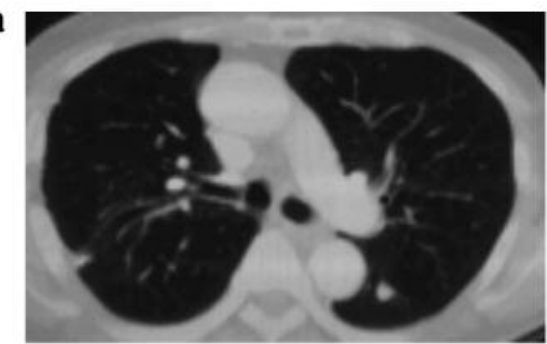

b

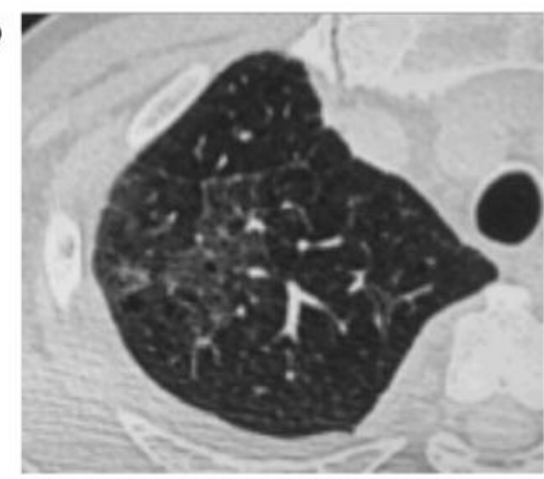

c

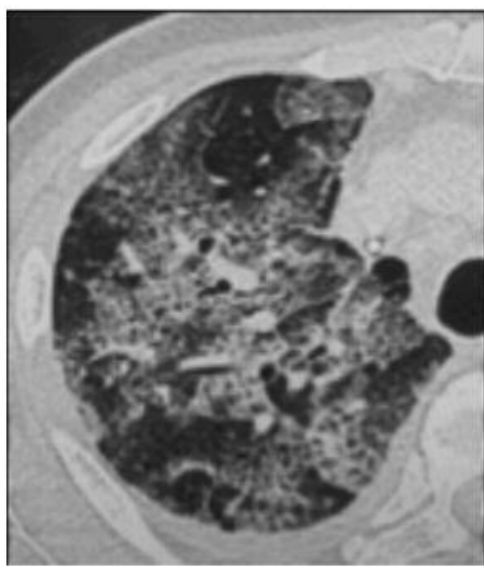

Figure 1. Computed tomography imaging findings for case 1. (a) The image shows pulmonary lesions before treatment, $(b)$ after 14 days of treatment with nivolumab, $(c)$ and after 23 days of treatment with nivolumab. Carcinomatous lymphangiosis can be clearly seen in the image obtained on day 23.

treated with immune checkpoint inhibitors showed accelerated tumour growth after treatment compared to that observed during the previous therapy for several types of neoplasms (6). The factor associated with this rapid progression was older age (>65 years); patients with hyperprogression showed a worse overall survival (6). Although no study has reported RPD in patients with RCC, this condition is possible. In case 2, the patient developed paraplegia owing to the rapid progression of vertebrae metastases. In cases 1 and 3, tumour growth was relatively quicker than the reported average in the phase of progressive disease before nivolumab therapy. These patients

had critical lesions that may have been related to symptomatic events or the high tumour burden with the high tumour growth rate. In addition, these patients received nivolumab in the lateline setting. These factors may be associated with RPD; however, additional patients need to be examined to re-analyze predictive factors.

We encountered 3 cases of RPD after nivolumab therapy for metastatic RCC. Clinicians should be aware of the possibility of RPD during the initial phase of nivolumab therapy, that may result in discontinuation of treatment, especially in the late-line setting. Screening for metastases to the central nervous system may be important to decrease the risk of RPD.

\section{Conflicts of Interest}

T. Kondo received honorarium from Ono Pharmaceutical and Bristol Myers Squibb. The other authors declare no competing interests.

\section{Acknowledgements}

The Authors would like to thank Editage (www.editage.jp) for English language editing.

\section{References}

1 Motzer RJ, Escudier B, McDermott DF, George S, Hammers HJ, Srinivas S, Tykodi SS, Sosman JA, Procopio G, Plimack ER, Castellano D, Choueiri TK, Gurney H, Donskov F, Bono P, Wagstaff J, Gauler TC, Ueda T, Tomita Y, Schutz FA, Kollmannsberger C, Larkin J, Ravaud A, Simon JS, Xu LA, Waxman IM, Sharma P, CheckMate 025 Investigators: Nivolumab versus everolimus in advanced renal-cell carcinoma. N Engl J Med 373: 1803-1813, 2015.

2 Sarfaty M, Moore A, Dudnik E and Peled N: Not only for melanoma. Subcutaneous pseudoprogression in lung squamouscell carcinoma treated with nivolumab: a case report. Medicine (Baltimore) 96: e5951, 2017.

3 Doherty MK, Jao K, Shepherd FA, Hazrati LN and Leighl NB: Central nervous system pseudoprogression in a patient treated with PD-1 checkpoint inhibitor. J Thorac Oncol 10: e100-e101, 2015.

4 Chiou VL and Burotto M: Pseudoprogression and immunerelated response in solid tumors. J Clin Oncol 33: 3541-3543, 2015.

5 Kanai O, Fujita K, Okamura M, Nakatani K and Mio T: Severe exacerbation or manifestation of primary disease related to nivolumab in non-small-cell lung cancer patients with poor performance status or brain metastases. Ann Oncol 27: 13541356, 2016.

6 Champiat S, Dercle L, Ammari S, Massard C, Hollebecque A, Postel-Vinay S, Chaput N, Eggermont A, Marabelle A, Soria JC and Ferté C: Hyperprogressive disease is a new pattern of progression in cancer patients treated by anti-PD-1/PD-L1. Clin Cancer Res 23: 1920-1928, 2017.

Received April 27, 2017

Revised May 25, 2017

Accepted May 26, 2017 